'Universidade Federal Rural de Pernambuco - UFRPE, R. Manuel de Medeiros, s/n, Dois Irmos, CEP 52171-900, Recife, PE, Brasil ${ }^{2}$ Bolsista de Doutorado CNPq 3̂nstituto Agronômico de Pernambuco - IPA, Recife, PE, Brasil

*autor correspondente \fclordao@uol.com.br

\title{
Efeito da suplementação com ácidos graxos associados à palma forrageira sobre a perfusão vascular endometrial em vacas Holandesas no pós-parto
}

\author{
Effect of the supplementation with fatty acids associated \\ with forage cactus on endometrial vascular perfusion in \\ postpartum Holstein cows
}

Fabiani Coutinho Lordão Guido ${ }^{1,2 *}$, Sebastião Inocêncio Guido ${ }^{3}$, Leonardo Fernandes de Alencar³, Júlio César Vieira de Oliveira ${ }^{3}$, Venézio Felipe dos Santos ${ }^{3}$, Joaquim Evêncio Neto

RESUMO: O objetivo deste estudo foi avaliar a influência do fornecimento de um composto de ácidos graxos (AG) no período de transição associado à dieta base com palma forrageira sobre a perfusão vascular uterina, escore de condição corporal, concentração de $\beta$-hidroxibutirato (BHB) e taxa de concepção pós-parto em vacas da raça Holandesa. $\mathrm{O}$ estudo foi realizado na Estação Experimental de São Bento do Una - EESBU /IPA, localizada no município de São Bento do Una, Pernambuco, Brasil, latitude 0831 ' 35 ' S, longitude 036 27' $34,8^{\prime \prime} \mathrm{W}$ e índice de temperatura e umidade (ITU) de 67,3 . Foram avaliadas 63 vacas no período de transição e mantidas em sistema semi-intensivo. As vacas aos 220 dias de gestação foram submetidas ao período pré-parto de 60 dias, tendo como dieta base $35 \mathrm{Kg}$ de palma forrageira, $40 \mathrm{Kg}$ de silagem de sorgo, além de complementação com $2 \mathrm{Kg}$ de concentrado proteico com $14 \%$ PB e adicionada de $5 \%$ de suplemento mineral nos primeiros 39 dias. Aos 21 dias antes do parto, foi alterada apenas a complementação proteica para $22 \%$ de PB. Sendo distribuídas em dois grupos experimentais (T1 e T2). No T1 ( $\mathrm{n}=30)$ as vacas receberam, no período de transição de 21 dias antes do parto, $200 \mathrm{~g}$ do composto de AG (gordura protegida), constituído pelo Ácido Linoleico, Ácido Linolênico e Ác. Linoleico Conjugado (Megalac ${ }^{\circledR}$-E, Vaccinar) adicionado ao concentrado proteico. OT2 ( $\mathrm{n}=33$ ) foi constituído por vacas que receberam apenas o concentrado proteico fornecido para o $\mathrm{T} 1$, sem adição do Megalac-E. Todas as vacas foram submetidas à avaliação do escore de condição corporal (ECC) na escala de 1 a 5 . Sendo avaliadas no início do período de transição aos 21 dias antes do parto e ao final do período voluntário de espera (PVE) aos 40 dias pós-parto. Para o monitoramento de distúrbios metabólicos, foi realizada em todas as vacas a dosagem das concentrações sanguíneas de BHB, sendo as dosagens realizadas no início do período de transição e ao final do PVE. Os exames ultrassonográficos foram realizados com aparelho de ultrassom na frequência de $6,0 \mathrm{MHz}$, utilizando transdutor endorretal linear no modo Doppler colorido para avaliação da perfusão vascular endometrial (PVENDO), sendo atribuídos escores de 1 a 4. Imediatamente após as avaliações, as vacas (T1 e T2) foram submetidos a protocolo de IATF, sendo o diagnóstico de prenhez realizado 30 dias após. Os dados foram submetidos à análise de variância ANOVA e ao teste Z a 5\%. Quanto aos resultados de ECC, não houve diferença $(p>0,05)$ entre os grupos (T1 a T2), nem tampouco em relação aos períodos pré e pós-parto. Em relação ao $\mathrm{BHB}$, as concentrações médias não evidenciaram diferença ( $\mathrm{p}>0,05)$ entre os grupos (T1 e T2) no pré-parto; todavia, no pós-parto, apresentaram diferença $(p<0,05)$, sendo as concentrações do T1 inferiores. $\mathrm{Na}$ avaliação da PVENDO, foram evidenciados no $\mathrm{T} 1$ os escores $1(26,7 \%), 2(56,7 \%), 3(6,6 \%)$ e $4(10,0 \%)$; sendo no T2 registrados os escores $1(18,2 \%), 2(39,4 \%), 3(30,3 \%)$ e $4(12,1 \%)$. A taxa de concepção foi mais elevada $(\mathrm{p}<0,05)$ nas vacas do T1 $(63,3 \%)$ em relação ao T2 $(42,4 \%)$. Portanto, conclui-se que a suplementação de vacas leiteiras com gordura protegida durante a primeira fase do período de transição, não influenciou a manutenção do ECC. Porém, os animais que receberam esta suplementação apresentaram resultados satisfatórios quanto aos valores de BHB e da taxa de concepção no pós-parto. Bem como os animais que receberam apenas o concentrado proteico apresentaram maior proporção de animais com escore 3 ao Doppler colorido, apresentando diagnóstico sugestivo de endometrite subclínica.

PALAVRAS-CHAVE: Bovino, perfusão vascular, endometrite, suplementação, ácidos graxos. 


\begin{abstract}
The objective of this study was to evaluate the influence of the supply of a fatty acid (FA) compound in the transition period associated with the diet with spineless cactus on uterine vascular perfusion, body condition score, $\beta$-hydroxybutyrate concentration (BHB) and rate of postpartum conception in Holstein cows. The study was carried out at the Experimental Station of São Bento do Una - EESBU/IPA, located in the city of São Bento do Una, Pernambuco, Brazil, latitude 0831 '35' 'S, longitude 03627 '34.8 “ $W$ and temperature- humidity index (THI) of 67.3. Sixty-three cows were evaluated in the transition period and maintained in a semi-intensive system. Cows at 220 days of pregnancy were submitted to the dry period of 60 days, with $35 \mathrm{~kg}$ of forage cactus, $40 \mathrm{~kg}$ of sorghum silage, as well as $2 \mathrm{~kg}$ protein concentrated with $14 \%$ PB and added $5 \%$ mineral supplement in the first 39 days. At 21 days prepartum, protein complementation was altered only for $22 \% \mathrm{CP}$. They are distributed in two experimental groups (T1 and T2). In the T1 $(n=30)$ the cows were received in the transition period of 21 days before calving $200 \mathrm{~g}$ of the compound of FA (protected fat), constituted by linoleic acid, linolenic acid and conjugated linoleic acid (Megalac ${ }^{\circledR}-E$, Vaccines) added to protein concentrate. $T 2(n=33)$ consisted of cows that received only the protein concentrate supplied to T1, without the addition of Megalac-E. All cows were submitted to the assessment of the body condition score (BCS) on the scale of 1 to 5. They were evaluated at the beginning of the transition period at 21 days before calving and at the end of the voluntary waiting period (VWP) at 40 days postpartum. For the monitoring of metabolic disorders, the blood concentrations of
\end{abstract}

BHB were measured in all cows, and the dosages were performed at the beginning of the transition period and at the end of the PVE. Ultrasound examinations were performed using a $6.0 \mathrm{MHz}$ ultrasound instrument using a color Doppler linear transrectal probe to assess endometrial vascular perfusion (PVENDO), with scores ranging from 1 to 4. Immediately after the evaluations, cows (T1 and T2) were submitted to the IATF protocol, and the diagnosis of pregnancy was performed 30 days later. Data were submitted to analysis of variance ANOVA and $Z$ test at $5 \%$. Regarding the $E C C$ results, there was no difference ( $p>0.05$ ) between the groups (T1 to T2), nor in relation to the pre and postpartum periods. Regarding the $B H B$, the mean concentrations did not show any difference ( $p>0.05$ ) between the groups (T1 and T2) in prepartum; however, postpartum presented a difference $(p<0.05)$, with lower concentrations in T1. In the evaluation of the PVENDO, 1 (26.7\%), $2(56.7 \%), 3(6.6 \%)$ and $4(10.0 \%)$ scores were evidenced in $\mathrm{T1}$; (18.2\%), 2 (39.4\%), 3 (30.3\%) and $4(12.1 \%)$ were recorded in T2. Conception rate was higher $(p<0.05)$ in $T 1$ cows $(63.3 \%)$ than T2 (42.4\%). Therefore, it was concluded that the supplementation of protected fat dairy cows during the first phase of the transition period did not influence the maintenance of BSC. However, the animals that received this supplementation presented satisfactory results regarding $\mathrm{BHB}$ values and conception rate in the postpartum period. Also, the animals that received only the protein concentrate presented a higher proportion of animals with color Doppler score 3, showing suggestive diagnosis of subclinical endometritis.

KEYWORDS: Cattle, vascular perfusion, endometritis, supplementation, fatty acids.

\section{Introdução}

O restabelecimento da ciclicidade e a fertilidade pós-parto em vacas leiteiras são fundamentais para um bom desempenho dos rebanhos. Neste contexto, os distúrbios metabólicos constituem os principais obstáculos a serem superados no período de transição (DRACKLEY, 1999; LEBLANC et al., 2006). O balanço energético negativo (BEN) ocorre independente da condição corporal da vaca (DRACKLEY, 1999; FERREIRA, 2010), entretanto, sua intensidade será maior se o suprimento da demanda energética não for suficientemente fornecido no pré-parto (ARTUNDUAGA et al., 2011). Geralmente, bovinos leiteiros são alimentados com quantidades moderadas de ácidos graxos de cadeia longa (AGCL) com o objetivo de aumentar a produção e o crescimento; todavia, o interesse pela adição de compostos energéticos na dieta de vacas leiteiras tem sido concentrado na eficiência reprodutiva, imunidade e produção (SANTOS et al., 2008; ARTUNDUAGA et al., 2010; SILVESTRE et al., 2011). No entanto, o monitoramento de doenças metabólicas de vacas leiteiras pode propiciar a implementação de protocolos que visem à correção de falhas de manejo e à diminuição do impacto negativo destes distúrbios no pós-parto (SILVESTRE et al., 2011; ALVARENGA et al., 2015; LUKAS et al., 2015). A utilização de suplemento composto por ácidos graxos (AG) polinsaturados pode contribuir com a correção da densidade energética necessária para vacas leiteiras neste período, influenciar o metabolismo de hormônios reprodutivos, o retorno à ciclicidade e a diminuição de doenças pós-parto (JUCHEM et al., 2010; Silva et al., 2014; GUIDO et al., 2016). Outro aspecto importante que se deve considerar é que as dietas básicas fornecidas a vacas leiteiras, geralmente, contêm apenas $2 \%$ de AGCL de origem vegetal, predominantemente, polinsaturados (SANTOS et al., 2008). Todavia, alguns alimentos podem naturalmente complementar a demanda energética da dieta de vacas leiteiras, como é o caso da palma forrageira (SANTOS et al., 2006; MONTEIRO et al., 2018). Deve-se considerar também que o mecanismo imunocelular de defesa associado a fatores quimiotáxicos e a hemodinâmica uterina podem sofrer influência do BEN (GRUNERT et al., 2005) e este poderá ser mitigado pela suplementação energética (ARTUNDUAGA et al., 2011). Portanto, a suplementação com composto energético associado à palma forrageira poderá diminuir a ocorrência de enfermidades pós-parto como a endometrite subclínica. 
Sendo de relevância o conhecimento da perfusão vascular endometrial para o diagnóstico da endometrite subclínica através da ultrassonografia no modo Doppler (GINTHER, 2007; GUIDO et al., 2019). Neste sentido, o objetivo deste trabalho foi avaliar a influência do fornecimento de um composto de ácidos graxos no período de transição associado à dieta base com palma forrageira sobre a perfusão vascular uterina, escore de condição corporal, concentração de $\beta$-hidroxibutirato (BHB) e taxa de concepção pós-parto em vacas da raça Holandesa.

\section{Material e Métodos}

O estudo foi realizado na Estação Experimental de São Bento do Una (EESBU), pertencente ao Instituto Agronômico de Pernambuco - IPA, localizada no município de São Bento do Una, Pernambuco, Brasil, latitude 08 31'35" S, longitude $03627^{\prime} 34,8^{\prime \prime} \mathrm{W}$ e índice de temperatura e umidade (ITU) de 67,3 para o período. Foram avaliadas vacas da raça Holandesa ( $\mathrm{n}=63$ ), no período de transição, três semanas antes e três semanas após o parto. As vacas, aos 220 dias de gestação, foram submetidas à secagem e ao período pré-parto de 60 dias, mantidas em sistema intensivo, tendo como dieta base $35 \mathrm{Kg}$ de palma forrageira (Opuntia spp.) contendo $12 \%$ de matéria seca (MS) $=4,2 \mathrm{Kg}, 40 \mathrm{Kg}$ de silagem de sorgo (Sorghum bicolor(L.) Moench) contendo 32\% de MS =12,8 Kg, além de complementação com $2 \mathrm{Kg}$ de concentrado proteico contendo $14 \%$ de proteína bruta $(\mathrm{PB})$ e $98 \%$ de $\mathrm{MS}=1,96 \mathrm{Kg}$, adicionada de $5 \%$ de suplemento mineral nos primeiros 39 dias. No período de transição, aos 21 dias antes do parto, foi alterada apenas a complementação proteica para $22 \%$ de $\mathrm{PB}$, sendo as vacas distribuídas em dois grupos experimentais (T1 e T2). No T1 $(\mathrm{n}=30)$, as vacas receberam, no período de transição, $200 \mathrm{~g}$ do composto de ácidos graxos (gordura protegida), constituído pelo Ácido Linoleico, Ácido Linolênico e Ác. Linoleico Conjugado (Megalac ${ }^{\circledR}-\mathrm{E}$, Vaccinar) adicionado ao concentrado proteico. O T2 $(n=33)$ foi constituído por vacas que receberam apenas o mesmo concentrado proteico fornecido para o T1, sem adição do Megalac-E.

Todas as vacas de ambos os grupos experimentais (T1 e T2) foram submetidas à avaliação do escore de condição corporal (ECC) realizada seguindo metodologia preconizada por Edmonson et al. (1989) em uma escala de 1 (muito magra) a 5 (obesa). Sendo, avaliadas no início do período de transição $21^{\circ}$ dia antes do parto e ao final do período voluntário de espera (PVE) aos 40 dias pós-parto.

Para o monitoramento de distúrbios metabólicos, foi realizada em todas as fêmeas de ambos os grupos (T1 e T2) a determinação das concentrações sanguíneas de $\beta$-Hidroxibutirato (BHB) com a utilização do medidor eletrônico Ketovet ${ }^{\circledR}$, sendo considerada como ponto de corte a concentração de $1,2 \mathrm{mmol} / \mathrm{mL}$ de acordo com Iwersen et al. (2013). A análise foi realizada através de leve punção com agulha estéril, realizada na extremidade da cauda de cada animal após antissepsia, sendo a gota sanguínea captada por capilaridade da fita de leitura e o resultado obtido em 5 segundos. A primeira leitura foi realizada no $21^{\circ}$ dia antes do parto e a segunda, ao final do PVE aos 40 dias pós-parto.

Para diagnóstico de endometrite subclínica, foi utilizada a avaliação através do escore de perfusão vascular endometrial (PVENDO), sendo atribuídos escores de 1 a 4 de acordo com Ginther (GUIDO et al., 2019). Os exames ultrassonográficos foram realizados utilizando-se um aparelho de ultrassom (Mindray $\mathrm{M}-5^{\circledR}$ ) equipado com transdutor endorretal linear na frequência de 6,0MHz no modo Doppler colorido, obtendo-se imagens no plano sagital longitudinal do terço médio de ambos os cornos uterinos. Os exames foram realizados pelo mesmo operador e as imagens, armazenadas na memória do equipamento e, posteriormente, avaliadas por dois técnicos habilitados.

Após as avaliações, todas as fêmeas de ambos os grupos experimentais (T1 e T2) foram submetidas a protocolo de IATF de acordo com Guido et al. (2012) e, posteriormente, no $30^{\circ}$ dia após a IATF, foram submetidas ao diagnóstico de prenhez através de palpação transretal auxiliada pela ultrassonografia.

Os dados foram submetidos à análise de variância ANOVA e ao teste $\mathrm{Z}$ para diferenças entre duas proporções a 5\%.

Este estudo foi submetido e aprovado pela comissão de ética no uso de animais (CEUA) da UFRPE (Licença $n^{\circ}$ 092/2017).

\section{Resultados e Discussão}

Os resultados de escore de condição corporal (ECC) registrados na Tabela 1 evidenciaram que não houve diferença $(\mathrm{p}>0,05)$ entre os grupos experimentais (T1 a T2), nem tampouco em relação aos períodos pré e pós-parto. Certamente, a complementação energética pela palma forrageira utilizada na dieta base fornecida às vacas de ambos os grupos experimentais ( $\mathrm{T} 1 \mathrm{e} \mathrm{T} 2$ ) pode ter sido responsável pela manutenção do ECC dos animais deste experimento. Ao contrário do reportado por Silvestre et al. (2011) que apenas obtiveram ganho de ECC em 37\% das vacas aos 43 dias pós-parto quando utilizaram como fonte de suplementação de AG o óleo de palma. Embora os efeitos deletérios do BEN sejam bem estabelecidos com a queda de ECC e mobilização das reservas corporais no pós-parto imediato, conforme relatam Drackley (1999) e Dann et al. (2006), pode-se inferir que a palma forrageira tenha sido eficiente para atenuar os efeitos negativos do BEN, tendo em vista as vacas de ambos os grupos (T1 e T2) não terem apresentado perda significativa de ECC. Estes resultados corroboram os de

Tabela 1. Parâmetros de escore de condição corporal (ECC), concentrações de $\beta$-hidroxibutirato (BHB) e Taxa de concepção em vacas Holandesas suplementadas no período de transição com composto de ácidos graxos (T1) e não suplementadas (T2). Estação Experimental de São Bento/IPA, Pernambuco, Brasil.

\begin{tabular}{|c|c|c|c|c|c|c|}
\hline \multirow{2}{*}{ Grupos } & \multirow{2}{*}{$N^{0}$ de vacas } & \multicolumn{2}{|c|}{ ECC } & \multicolumn{2}{|c|}{ ВHB } & \multirow{2}{*}{$\begin{array}{c}\text { Taxa de } \\
\text { Concepçãc }\end{array}$} \\
\hline & & Pré-parto & Pós-parto & Pré-parto & Pós-parto & \\
\hline $\mathbf{T 1}$ & 30 & 2,55 & 2,30 & 1,26 & $0,97^{a}$ & $63,3^{a}$ \\
\hline T2 & 33 & 2,50 & 2,24 & 1,29 & $1,69^{\mathrm{a}}$ & $42,4^{\mathrm{a}}$ \\
\hline
\end{tabular}

${ }^{\mathrm{a}} \mathrm{p}<0,05$. 
Silvestre et al. (2011) que relatam melhores resultados quando associaram diferentes fontes energéticas na dieta de vacas leiteiras no período de transição. Pode-se também sugerir que houve um BEN brando, tendo em vista, neste experimento, as vacas não apresentarem perda significativa de ECC, sendo possível considerá-lo como indicativo da condição energética, conforme relatam Silvestre et al. (2011).

Em relação ao indicador metabólico BHB, os resultados das concentrações médias não evidenciaram diferença $(p>0,05)$ entre os grupos (T1 e T2) no pré-parto e, no pós-parto, apresentaram diferença $(p<0,05)$, sendo as concentrações do T1 inferiores às do T2 (Tabela 1). Estes resultados divergem dos registrados por Silvestre et al. (2011), que obtiveram acréscimo nas concentrações do BHB no pós-parto, mesmo suplementando as vacas com diferentes fontes de $\mathrm{AG}$ no período de transição, entretanto sem prejuízos para concepção. Bem como os obtidos por Alvarenga et al. (2015) quando avaliaram o perfil metabólico de vacas leiteiras no período de transição. Porém, em controvérsia, Ribeiro et al. (2013) registraram, em vacas com doenças clínicas puerperais, menores concentrações de BHB que em suas companheiras de rebanho sem doenças clínicas; no entanto, vacas com concentrações elevadas de ácidos graxos não esterificados (AGNE), e, portanto, sob forte influência do BEN, apresentaram concentrações de BHB aumentadas (RIBEIRO et al., 2013). Contudo, neste estudo, a melhor taxa de concepção foi registrada no T1 (Tabela 1), nas vacas que apresentaram menor concentração de BHB no pós-parto. O que respalda a utilização da dosagem do BHB como indicador de distúrbios metabólicos, considerando concentrações inferiores a $1,20 \mathrm{mmol} / \mathrm{L}$ de acordo com Iwersen et al. (2013). Concentrações elevadas do BHB no pós-parto são relatadas por diversos autores (LEBLANC et al., 2006; ALVARENGA et al., 2015) e estão relacionadas à mobilização AGNE e intensidade do BEN (OSPINA et al., 2010). Entretanto, deve-se considerar que vacas que apresentam menor risco de distúrbios metabólicos apresentam também melhor desempenho reprodutivo pós-parto (SANTOS et al., 2008; SILVESTRE et al., 2011; RIBEIRO et al., 2013).

$\mathrm{Na}$ avaliação do escore de perfusão vascular endometrial (PVENDO) com Doppler colorido de acordo com critérios estabelecidos por Ginther (2007), foram evidenciados animais apresentando os escores de 1 a 4 (Tabela 2) em ambos os grupos experimentais (T1 e T2). Sendo os resultados para o escores 2 e 3 inferiores $(p<0,05)$ no T1. Todavia, houve uma maior proporção de vacas apresentando o escore 3 de PVENDO no T2 (Tabela 2). Fato que sugere uma maior prevalência de endometrite subclínica (ES) neste grupo, conforme sugerem Guido et al. (2019), utilizando o mesmo padrão de escores de perfusão vascular endometrial como método diagnóstico dessa enfermidade. Estes resultados corroboram os achados de Debertolis et al. (2016) que associaram o aumento da perfusão vascular do endométrio com a ocorrência de endometrite induzida em vacas. Bem como os de Jordan et al. (2009) que associaram positivamente a perfusão vascular com eventos que promovem o aumento do fluxo sanguíneo uterino. Entretanto, deve-se salientar que se esperava uma menor ocorrência de doenças uterinas nos animais suplementados com o composto de AG (T1), conforme relatam diversos autores (SANTOS et al., 2008; RIBEIRO et al., 2013), principalmente a ocorrência de quadros subclínicos como as endometrites, que foram registradas em apenas 13,4\% em vacas suplementadas (RIBEIRO et al., 2013). Considerando que as endometrites constituem as causas mais relevantes de atraso da ciclicidade e baixa taxa de concepção em vacas leiteiras (KASIMANICKAM et al., 2004; SHELDON et al., 2009), alternativas de diagnósticos de rápida execução devem sempre constituir a prioridade no acompanhamento de reprodutivo de rebanhos leiteiros. Daí a opção de utilização de um método não invasivo e de elevada sensibilidade como a ultrassonografia no modo Doppler colorido para avaliação das endometrites, conforme preconizam Debertolis et al. (2016) e Guido et al. (2019).

Quanto às taxas de concepção registradas após a IATF, o T1 apresentou resultado superior $(\mathrm{p}<0,05)$ ao T2 (Tabela 1) aos 30 dias. Contudo, quando se considera os escores de PVENDO, registra-se que houve uma maior proporção de vacas prenhes dentre aquelas que foram classificadas com os escores 1 e 2 (Figura 1). Estes resultados se assemelham aos obtidos por Guido et al. (2019), quando utilizaram o mesmo método de avaliação da perfusão vascular endometrial. Ademais, deve-se

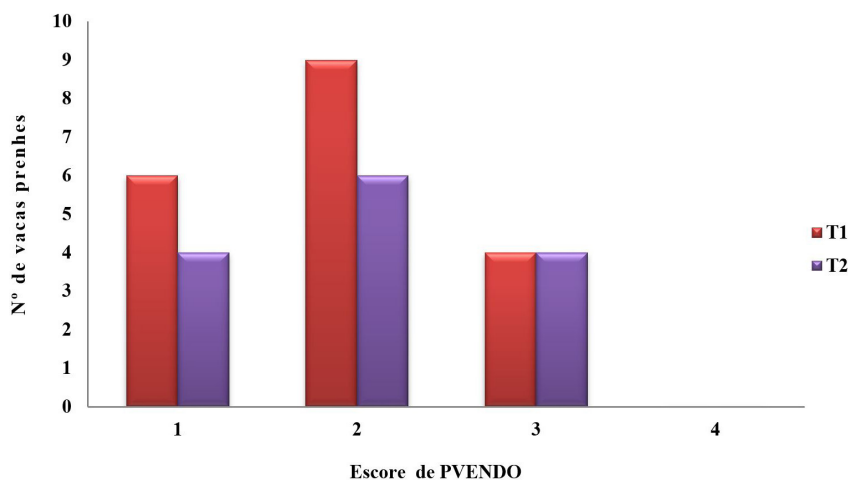

Figura 1. Distribuição de prenhez de acordo com o escore de perfusão vascular endometrial e em vacas Holandesas suplementadas no período de transição com composto de ácidos graxos (T1) e não suplementadas (T2). Estação Experimental de São Bento/IPA, Pernambuco, Brasil.

Tabela 2. Escore de perfusão vascular endometrial pós-parto em vacas Holandesas suplementadas no período de transição com composto de ácidos graxos (T1) e não suplementadas (T2). Estação Experimental de São Bento/IPA, Pernambuco, Brasil.

\begin{tabular}{|c|c|c|c|c|c|}
\hline \multirow{3}{*}{ Grupos } & \multirow{3}{*}{$N^{0}$ de vacas } & \multicolumn{4}{|c|}{ Perfusão Vascular Endometrial - Escore } \\
\hline & & 1 & 2 & 3 & 4 \\
\hline & & $\mathbf{N}(\%)$ & $\mathbf{N}(\%)$ & $\mathbf{N}(\%)$ & $\mathbf{N}(\%)$ \\
\hline $\mathbf{T 1}$ & 30 & $8(26,7)$ & $17(56,7)^{\mathrm{a}}$ & $2(6,6)^{a}$ & $3(10,0)^{\mathrm{a}}$ \\
\hline $\mathbf{T} 2$ & 33 & $6(18,2)$ & $13(39,4)^{a}$ & $10(30,3)^{a}$ & $4(12,1)^{\mathrm{a}}$ \\
\hline
\end{tabular}

${ }^{\mathrm{a}} \mathrm{p}<0,05$. 
considerar também a influência do protocolo de IATF, tendo em vista as vacas classificadas com o escore 2 de PVENDO terem apresentado uma maior taxa de concepção para ambos os grupos (T1 e T2), conforme registrado (Figura 1). Neste sentido, a utilização de ferramentas como a ultrassonografia e a avaliação do ECC para seleção de vacas leiteiras para programas tem resultado em ganhos de eficiência, conforme respaldam Guido et al. (2017), Viana e Siqueira (2017) e Guido et al. (2018).

\section{Conclusão}

A suplementação de vacas leiteiras com gordura protegida durante a primeira fase do período de transição não influenciou a manutenção do ECC. Porém os animais que receberam esta suplementação apresentaram resultados satisfatórios quanto aos valores de BHB e da taxa de concepção no pós-parto. Bem como os animais que receberam apenas o concentrado proteico apresentaram maior proporção de animais com escore 3 ao Doppler colorido, apresentando diagnóstico sugestivo de endometrite subclínica.

\section{Agradecimentos}

Ao CNPq pelo apoio financeiro e ao IPA pela cooperação na execução da pesquisa.

\section{Referências}

ALVARENGA, E. A. et al. Avaliação do perfil metabólico de vacas da raça Holandesa durante o período de transição. Pesquisa Veterinária Brasileira, Rio de Janeiro, v. 35, n. 3, p. 281-290, 2015.

ARTUNDUAGA, M. A. T. et al. Incidência de doenças no pós-parto de primíparas da raça Holandesa alimentadas com diferentes fontes energéticas durante o período de transição. Arquivo Brasileiro de Medicina Veterinária e Zootecnia, Belo Horizonte, v. 63, n. 3, p. 616-623, 2011.

ARTUNDUAGA, M. A. T. et al. Primeira onda folicular e ovulação de vacas primíparas da raça Holandesa alimentadas com diferentes fontes energéticas durante o período de transição. Arquivo Brasileiro de Medicina Veterinária e Zootecnia, Belo Horizonte, v. 62, n. 1, p. 116-123, 2010.

DANN, H. M. et al. Diets during far-off and close-up dry periods affect periparturient metabolism and lactation in multiparous cows. Journal of Dairy Science, Champaign, v. 89, n. 9, p. 3563-3577, 2006.

DEBERTOLIS, L. et al. Effects if induced endometritis on uterine blood flow in cows as evaluated by transrectal Doppler sonography. Journal of Veterinary Science, Suwŏn-shi, v. 17, n. 2, p. 189-197, 2016.

DRACKLEY, J. K. Biology of dairy cows during the transition period: the final frontier? Journal of Dairy Science, Champaign, v. 82, n. 11, p. 2259-2273, 1999.

EDMONSON, A. J. et al. A body condition scoring chart for Holstein dairy cows. Journal of Dairy Science, Champaign, v. 72, p. 68-78, 1989.

FERREIRA, A. M. Reprodução da fêmea bovina: fisiologia aplicada e problemas mais comuns (causas e tratamentos). 1. ed. Juiz de Fora: Editar, 2010. 420 p.
GINTHER, O. J. Ultrasonic imaging and animal reproduction: color-doppler ultrasonography. v. 4. Wisconsin: Equiservices Publishing, 2007. 258 p.

GRUNERT, E. et al. Patologia e clínica da reprodução dos animais domésticos. São Paulo: Livraria Varela, 2005. 551 p.

GUIDO, F. C. L. et al. Incidência de distúrbios reprodutivos em vacas da raça holandesa criadas em condições de semiárido. Ciência Veterinária nos Tropicos, Recife, v. 19, n. 1, p. 52-57, 2016.

GUIDO, F. C. L. et al. Utilização do dispositivo Metricheck e da ultrassonografia para seleção de vacas leiteiras em programas de inseminação artificial em tempo fixo. Revista Brasileira de Reprodução Animal, Belo Horizonte, v. 41, n. 3, p. 678-682, 2017.

GUIDO, F.C.L. et al. Utilização da perfusão vascular endometrial, ultrassonografia modo $\mathrm{B}$ e citologia para o diagnóstico de endometrite subclínica em vacas Holandesas. Indian Journal of Dairy Science, New Delhi, 2019. No prelo.

GUIDO, S. I. et al. Influence of parity category on conception rate of Holstein females submitted to timed artificial insemination in semiarid conditions. Animal Reproduction, Belo Horizonte, v. 15, p. 1126, 2018.

GUIDO, S. I. et al. Reproductive efficiency of Holstein cows submited to fixed time artificial insemination in semiarid regions. Reproduction in Domestic Animals, Oxford, v. 47, p. 424, 2012.

IWERSEN, M. et al. Comparison of 2 electronic cowside tests to detect subclinical ketosis in dairy cows and the influence of the temperature and type of blood sample on the test results. Journal of Dairy Science, Champaign, v. 96, n. 12, p. 7719-7730, 2013.

JORDAN, A. et al. Genital blood flow and endometrial gene expression during the preovulatory period after prostaglandin F2 $\alpha \mathrm{n}$ - Induced luteolysis in different luteal phases in cows. The Journal of Reproduction and Development, Fuchū, v. 55, n. 3, p. 309-315, 2009.

JUCHEM, S. O. et al. Supplementation with calcium salts of linoleic and trans-octadecenoic acids improves fertility of lactanting daury cows. Reproduction in Animals Domestics, Oxford, v. 45, p. 55-62, 2010.

KASIMANICKAM, R. et al. Endometrial cytology and ultrasonography for the detection of subclinical endometritis is postpartum dairy cows. Theriogenology, Milan, v. 62, n. 1, p. 9-23, 2004.

LEBLANC, S. J. et al. Major advances in disease prevention in dairy cattle. Journal of Dairy Science, Champaign, v. 89, n. 4, p. 1267-1279, 2006.

LUKAS, J. M. et al. A study of methods for evaluating the success of the transition period in early-lactation dairy cows. Journal of Dairy Science, Champaign, v. 98, n. 1, p. 250-262, 2015.

MONTEIRO, C. C. F. et al. A new cactus variety for dairy cows in areas infested with Dactylopius opuntiae. Animal Production Science, Melbourne, v. 59, n. 3, p. 479-485, 2018. http://dx.doi. org/10.1071/AN17256.

OSPINA, P. A. et al. Associations of elevated nonesterified fatty acids and $\beta$-hydroxybutyrate concentrations with early lactation reproductive performance and milk production in transition dairy cattle in the northeastern United States. Journal of Dairy Science, Champaign, v. 93, n. 4, p. 1596-1603, 2010.

RIBEIRO, E. S. et al. Prevalence of periparturient diseases and effects on fertility of seasonally calving grazing dairy cows supplemented 
with concentrates. Journal of Dairy Science, Champaign, v. 96, n. 9, p. 5682-5697, 2013.

SANTOS, D. C. et al. Manejo e utilização da palma forrageira (Opuntia e Nopalea) em Pernambuco. Recife: IPA, 2006. 48 p. Documentos, n. 30.

SANTOS, J. E. P. et al. Long chain fatty acids of diet as factors influencing reproduction in cattle. Reproduction in Domestic Animals, Oxford, v. 43, p. 23-30, 2008.

SHELDON, I. M. et al. Mechanisms of infertility associated with clinical and subclinical endometritis in high producing dairy cattle. Reproduction in Domestic Animals, Oxford, v. 44, p. 1-9, 2009.
SILVA, J. A. et al. Utilização de gordura na dieta de vacas leiteiras. Pubvet, Londrina, v. 8, n. 17, p. 1772, 2014.

SILVESTRE, F. T. et al. Effects of differential supplementation of fatty acids during the peripartum and breeding period of Holstein cows: I. Uterine and metabolic responses, reproduction, and lacttion. Journal of Dairy Science, Champaign, v. 94, n. 1, p. 189-204, 2011.

VIANA, J. H. M.; SIQUEIRA, L. G. B. Current status, emerging technologies, and trends in veterinary ultrasonography applied to cattle reproduction. Spermova, Lima, v. 7, n. 2, p. 85-92, 2017.

Recebido: 07 fev. 2019 Aprovado: 16 mar. 2019 